\title{
A Comparative Molecular Dynamics, MM-PBSA and Thermodynamic Integration Study of Saquinavir Complexes with Wild-Type HIV-1 PR and L10I, G48V, L63P, A71V, G73S, V82A and I84V Single Mutants
}

\author{
Haralambos Tzoupis, ${ }^{*}+, \dot{\dagger}$ Georgios Leonis, ${ }^{* \dagger}$ Thomas Mavromoustakos, ${ }^{\dagger}$
} and Manthos G. Papadopoulos* ${ }^{* \dagger}$

${ }^{\dagger}$ Institute of Biology, Medicinal Chemistry and Biotechnology, National Hellenic Research Foundation, 48 Vas. Constantinou Ave., Athens 11635, Greece

${ }^{\ddagger}$ Department of Chemistry, National and Kapodistrian University of Athens, Panepistimioupolis Zographou 15771, Greece

Supporting Information

\begin{abstract}
A great challenge toward Acquired Immunodeficiency Syndrome (AIDS) treatment is to combat the HIV-1 virus. The major problem of drug resistance has kept the virus one step ahead of the medical community, and the call for more effective drugs remains as urgent as ever. Saquinavir, the first inhibitor against HIV-1 protease, offers the most extensive clinical data regarding resistance mutations. In this work, we examine L10I, G48V, L63P, A71V, G73S, V82A, and I84V single mutant HIV-1 PR strains in complexes with saquinavir to elucidate drug-protease interactions and dynamics. A comparative analysis of these mutations at the molecular level may lead to a deeper understanding of saquinavir resistance. The G48V mutation induces structural changes to the protease that reflect upon the drug's binding affinity, as shown by MM-PBSA and thermodynamic integration (TI) calculations $\left(\Delta \Delta G_{\mathrm{TI}}=0.3 \mathrm{kcal} / \mathrm{mol}\right.$; $\left.\Delta \Delta G_{\mathrm{MM}-\mathrm{PBSA}}=1.2 \mathrm{kcal} / \mathrm{mol}\right)$. It was shown that mutations, which increase the flexibility of the flaps (G48V, L63P, L10I) diminish binding. The preservation of hydrogen bonds of saquinavir with both the active site and flap residues in the wild-type and certain single mutants (A71V, V82A) is also crucial for effective inhibition. It was shown that mutations conferring major resistance (G48V, L63P, I84V) did not present these interactions. Finally, it was indicated that a water-mediated hydrogen bond between saquinavir and Asp29 in the active site (wild-type, A71V, G73S) facilitates a proper placement of the drug into the binding cavity that favors binding. Mutants lacking this interaction (G48V, V82A, I84V) demonstrated reduced binding affinities. This systematic and comparative study is a contribution to the elucidation of the drug resistance mechanism in HIV-1 PR.
\end{abstract}

\section{INTRODUCTION}

The spread of acquired immunodeficiency syndrome (AIDS) has proven a major clinical challenge as more than 33 million people are infected worldwide. ${ }^{1}$ For the past three decades, there has been extensive research of the human immunodeficiency virus (HIV) that has led to the discovery of several antiviral agents. According to the U.S. FDA, ${ }^{2}$ currently there are 30 commercially available anti-HIV drugs, divided into 16 reverse transcriptase (RT) inhibitors, nine protease (PR) inhibitors, one fusion inhibitor, one integrase inhibitor, one entry inhibitor, and two combination drugs. ${ }^{3}$ Although most treatment protocols had been relatively successful, the therapeutic benefits diminish over time due to the appearance of drug resistance. This eventually led to the introduction of a combinatorial regime involving $\mathrm{RT}$ and $\mathrm{PR}$ inhibitors, as opposed to monodrug therapy. The administration of such drug cocktails is called highly active antiretroviral therapy $(\text { HAART })^{4}$ and has resulted in a great decrease in HIV morbidity and mortality rates. ${ }^{5,6}$ Nevertheless, it became apparent that the effectiveness of HAART does not prevent treatment failure. This is mainly due to problems in HAART adherence and drug-drug interactions, while the emergence of drug-resistant viral strains remains the most important drawback. ${ }^{7,8}$

HIV-1 protease (HIV-1 PR) is an aspartic protease consisting of two identical chains, with 99 amino acids each. The active site of the protein comprises two aspartic acids (Asp) in positions $25 / 25^{\prime}$ as parts of two catalytic triads (Asp-ThrGly), common in most members of this family. ${ }^{9}$ The AspThr-Gly triplets are located at the bottom of a binding cleft, which is covered by two flaps (residues $44-55 / 44^{\prime}-55^{\prime}$; Figure 1). The flap region modulates the entrance of substrates to the active site cavity. ${ }^{10,11}$ The flaps have been also implicated in direct interactions with substrates, thus anchoring them firmly inside the binding cavity. ${ }^{12}$ Experimental and theoretical studies

Received: December 4, 2012

Published: February 5, 2013 

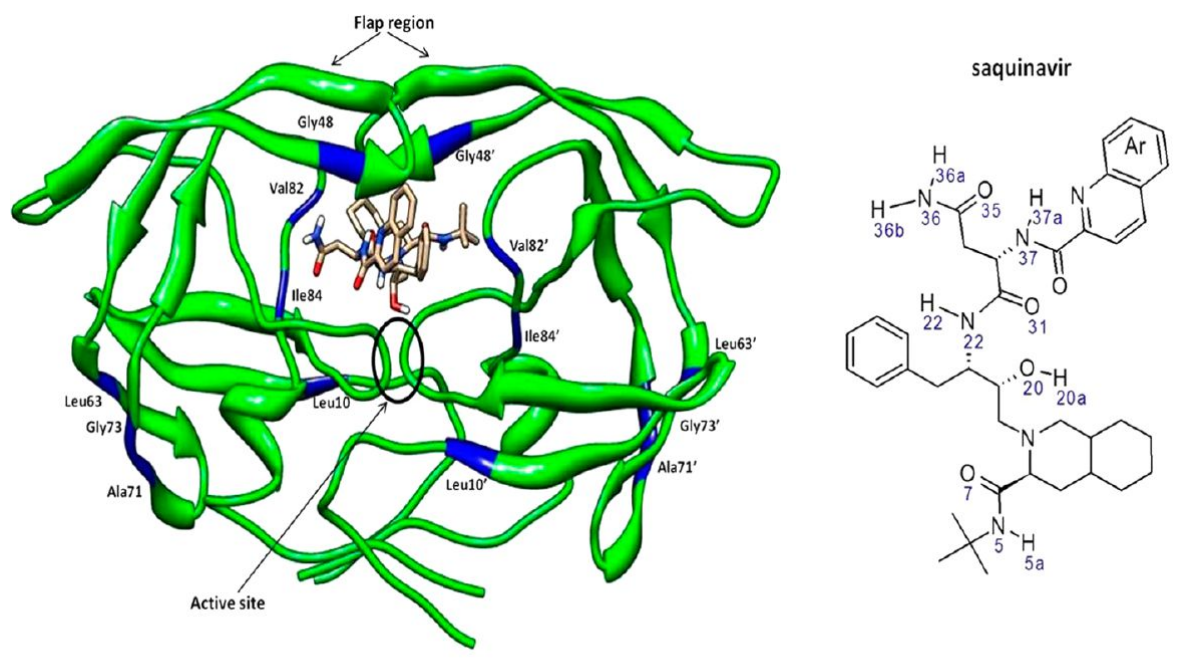

Gag-Pol HIV-1 PR cleavage site $\rightarrow$...LREDLAF-PQGKARE...

Figure 1. Representation of the saquinavir-HIV-1 PR complex (left) and the chemical structure of saquinavir (right). The positions of the seven single mutations are highlighted in blue. A part of the Gag-Pol precursor sequence, where cleavage occurs (Phe-Pro bond) is also shown.

revealed that the flaps in the apo form of HIV-1 PR are highly flexible and appear to be in equilibrium among three states, namely "closed," "open," and "semi-open"; 9,10,13 upon binding of a substrate or inhibitor, it has been shown that they adopt mainly a "closed" conformation. ${ }^{11,14}$

The emergence of drug resistant viral strains, in general, presents the following pattern. An initial, single mutation leads to the appearance of low level resistance, followed by the accumulation of mutations in the protease that produces strains with a high level of resistance against a specific inhibitor, as well as cross-resistance to other protease inhibitors (PIs). ${ }^{15,16}$ One of the potent, first-generation PIs is saquinavir (SQV, Figure 1), which acts as a peptidomimetic compound for HIV-1 PR's natural substrate. ${ }^{17}$ Actually, it has been characterized as a transition-state analog of the Phe-Pro amide bond in the GagPol precursor protein (Figure 1). ${ }^{18}$ Since saquinavir was the first PI approved, numerous studies have provided a vast amount of information to date regarding HIV-1 PR mutations that reduce susceptibility to this drug. One of the issues regarding saquinavir is the low oral bioavailability due to its lipophilic nature. ${ }^{19}$ To overcome this, high doses of the inhibitor are administered; however, this leads not only to serious side effects such as lipodystrophy and hypertension ${ }^{20}$ but also to increased drug resistance.

Following extensive clinical studies in patients, researchers have isolated drug-resistant HIV-1 strains, and the corresponding mutations have been identified. ${ }^{21}$ In this study, we focus on seven important saquinavir-related, single mutations in positions 10/10' (L10I), 48/48' (G48V), 63/63' (L63P), 71/ $71^{\prime}$ (A71V), 73/73' (G73S), 82/82' (V82A), and 84/84' $(\mathrm{I} 84 \mathrm{~V})$, as shown in Figure 1. The strength of this work lies in the comparison of several saquinavir-associated single mutants, something that has not been attempted to such an extent before. Importantly, analysis on many mutants enables a broad understanding of saquinavir resistance. ${ }^{21}$ Among these seven different mutation sites that have been identified, the mutation from Gly to Val in positions $48 / 48^{\prime}$ has the greatest impact to saquinavir resistance; ${ }^{22}$ this residue is located at the flap region of the enzyme, which is directly responsible for the entrapment of ligands into the active site.
The clinical data on the mutations merely reveal the site of each one, and do not provide information regarding the binding interactions of the drug. It is of particular importance to reveal the nature of resistance by studying on the molecular level the changes induced to the saquinavir-protease complexes upon different mutations and focus on the common patterns that may appear. Theoretical methods, such as molecular dynamics and free energy approaches (thermodynamic integration, MMPBSA), offer valuable insight regarding this.

Most computational studies, such as that by Ode et al., ${ }^{23}$ consider the impact a mutation has upon drug binding, usually in the vicinity of the mutation site. Also, most conformational analyse $^{24,25}$ look at the immediate area surrounding the drug and assess any changes in direct drug-protein interaction patterns, ${ }^{26,27}$ caused by the mutation. In this study, we consider the way mutations affect the entire protease structure and compare common features among mutants, as well as between mutants and the wild-type (wt). Conformational analysis and the study of interactions in the mutant complexes compared to the wt enzyme elucidate changes in the protease that may be crucial for effective binding. Here, we combine molecular dynamics (MD), molecular mechanics Poisson-Boltzmann surface area (MM-PBSA), and thermodynamic integration (TI) techniques to analyze the impact of single mutations on the binding affinity of saquinavir for HIV-1 PR. The estimation of the binding free-energy and its relative change (between wt and mutant) offers valuable information into drug-target relationships. The potency of a new inhibitor strongly depends on this thermodynamic potential. ${ }^{28,29}$

\section{METHODS}

In this section, we describe the procedures followed in our study. First, the construction of the complexes based on the crystal structure of the wt protease is described. The next step involves the setup of the systems for MD simulations, and finally we provide the specifics for the MM-PBSA and TI analyses.

II.1. Systems. We obtained the high resolution (1.16 A) crystal structure of wt HIV-1 PR in complex with saquinavir from the Brookhaven Protein Databank (PDB code: 3OXC). ${ }^{30}$ 
For consistency in our calculations, we used this protease structure as a template for the construction of the single mutant complexes. We substituted the wt amino acids in positions $10 /$ $10^{\prime}, 48 / 48^{\prime}, 63 / 63^{\prime}, 71 / 71^{\prime}, 73 / 73^{\prime}, 82 / 82^{\prime}$, and $84 / 84^{\prime}$ (Table $\mathrm{S} 1$ ), in order to create the respective single mutants. This has been done according to previous studies examining the effect of single mutations. ${ }^{31-33}$ The active site was considered monoprotonated in position 25 , in agreement with previous experimental and computational evidence. ${ }^{34,35}$ TI calculations were performed for the G48V mutation, and we substituted the Gly only in position 48 (chain A), while Gly48' remained unchanged. The TI method is highly sensitive to the number of different atoms between wt and mutated complexes; thus, for the method to produce accurate results, the systems must not have great changes.

II.2. Molecular Dynamics Simulations in Water. The complexes were subjected to unrestrained MD simulations in explicit solvent using the SANDER module of AMBER $11 .^{36}$ The ff99SB force field was used to represent the behavior of the protease. ${ }^{37}$ The parameters for saquinavir were developed with ANTECHAMBER (using the GAFF force field with AM1-BCC charges). ${ }^{38,39}$ All complexes were solvated in water (using the TIP3P water model), ${ }^{40}$ and truncated octahedral periodic boundary conditions have been applied (cutoff distance $8 \AA$ ). Minimization of the complexes was first performed by keeping the solute atoms constrained (with a harmonic force constant = $500 \mathrm{kcal} \mathrm{mol}^{-1} \AA^{-2}$ ) over 5000 steps, with the first 2500 steps employing the steepest descent method and the next 2500 steps with the conjugate gradient algorithm. A second 5000step minimization was performed with all atoms unrestrained. Next, the systems were gently heated under constant volume for $100 \mathrm{ps}$, with the gradual increase of the temperature from 0 to $300 \mathrm{~K}$, and were equilibrated under constant pressure for $100 \mathrm{ps}$. In both heating and equilibration procedures, a restraint of $10 \mathrm{kcal} \mathrm{mol}^{-1} \AA^{-2}$ has been applied to each solute. The constant-pressure equilibration has been prolonged for another 200 ps after all restraints had been removed. Finally, the MD simulations (total $20 \mathrm{~ns}$ each) were done in the NPT ensemble, using a Langevin dynamics temperature scaling (with collision frequency $\left.2 \mathrm{ps}^{-1}\right){ }^{41}$ All bonds involving hydrogen atoms were constrained to their equilibrium distance (in all steps) with the SHAKE algorithm, thus allowing for a time step of $2 \mathrm{fs}$ to be used. $^{42}$ For the analysis of the hydrogen bond (HB) interactions, the limitations were a $3.5 \AA$ cutoff for each donor-acceptor distance and a donor-hydrogen-acceptor angle cutoff of $120^{\circ}$. HB, RMSD, atomic fluctuations, and clustering calculations were performed with the ptraj program of AMBER.

II.3. Molecular Mechanics Poisson-Boltzmann Surface Area Calculations. MM-PBSA expresses the binding energy of a system (eq 1) as a sum of enthalpy $(H)$ and entropy (S) parts (eq 2). ${ }^{43}$ The enthalpy component is further decomposed in various contributions (eq 3, van der Waals, $\Delta E_{\mathrm{vdW}}$; electrostatic, $\Delta E_{\text {elec; }}$ nonpolar solvation, $\Delta G_{\mathrm{NP}} ;$ and polar solvation, $\Delta G_{\mathrm{PB}}$ ). The van der Waals and electrostatic components are calculated using molecular mechanics (MM), while the estimation of the electrostatic contribution to the solvation free energy is achieved with the Poisson-Boltzmann equation. The nonpolar contribution to the solvation energy is calculated with an empirical method based on the solvent accessible surface area (SASA), ${ }^{44}$ as shown in eq 4.

The binding free energy of the complex, for a given snapshot, is given by

$$
\Delta G_{\text {bind }}=G_{\text {complex }}-\left(G_{\text {receptor }}+G_{\text {ligand }}\right)
$$

where $G$ is the free energy of each system (complex or receptor or ligand) calculated as the average of free energies over the 20 ns trajectory.

$$
\begin{aligned}
& \Delta G_{\text {bind }}=\Delta H-T \Delta S \\
& \Delta H=\Delta E_{\text {elec }}+\Delta E_{\mathrm{vdW}}+\Delta G_{\mathrm{PB}}+\Delta G_{\mathrm{NP}} \\
& \Delta G_{\mathrm{NP}}=\gamma \mathrm{SASA}+\beta
\end{aligned}
$$

For the surface tension $\gamma$ and the offset $\beta$, the default values of $0.00542 \mathrm{kcal} \mathrm{mol}^{-1} \AA^{-2}$ and $0.92 \mathrm{kcal} \mathrm{mol}^{-1}$ were used, respectively. $\Delta G_{\mathrm{NP}}$ is computed via eq 4 , with the linear combinations of pairwise overlaps (LCPO) method. ${ }^{45}$

The HIV-1 PR binding cavity is highly hydrophobic, ${ }^{44}$ and according to previous theoretical studies ${ }^{46,47}$ the dielectric constants better suited to our calculations for the solvent and the solute are 80.0 and 1.0, respectively. The entropic component of eq 2 was calculated with normal-mode analysis. To model the water environment around the protein, MMPBSA strips the explicit water molecules and uses a parametrized implicit water model (PB). While enthalpy calculations were performed on 2000 frames of each trajectory, only 200 snapshots were used for the entropy estimation, to save computational time.

II.4. Thermodynamic Integration Calculations. Calculating the binding energy $(\Delta G)$ in protein complexes offers insights into the binding mechanism. However, the most important information when comparing complexes, such as wt and mutant proteases (in this case only for G48V), comes from the relative difference $(\Delta \Delta G)$ between the two states. ${ }^{48} \mathrm{TI}$ is a technique that calculates the relative energy difference from $\mathrm{MD}$ or Monte Carlo (MC) simulations and is based on the following scheme:

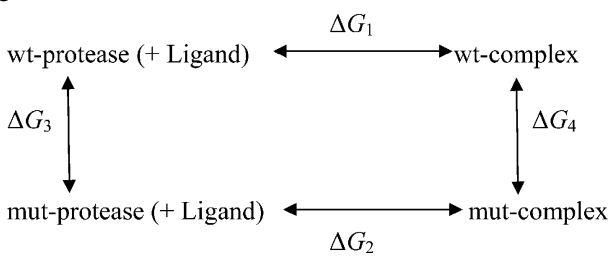

where

$$
\Delta \Delta G=\Delta G_{2}-\Delta G_{1}=\Delta G_{4}-\Delta G_{3}
$$

$\Delta G_{1}$ and $\Delta G_{2}$ represent the binding of a ligand to two different proteins (wt and mutated forms), while $\Delta G_{3}$ and $\Delta G_{4}$ represent transformations from one protein to the other, either being only solvated in water $\left(\Delta G_{3}\right)$ or when bound to a ligand $\left(\Delta G_{4}\right)$.

The TI method calculates the binding free energy based on the following equation: ${ }^{48}$

$$
\Delta G=\sum_{i=1}^{n} W_{i}\left(\frac{\partial V}{\partial \lambda}\right) \lambda_{i}
$$

where $\lambda$ is the coupling parameter and $V$ denotes the $\lambda$-coupling potential function. ${ }^{49}$ The values for $\lambda$ and their respective weights $\left(W_{i}\right)$ are assigned based on the Gaussian quadratic formula. ${ }^{50}$ For $\lambda=0$, the $V(0)$ potential function corresponds to the wt, and for $\lambda=1$, the $V(1)$ potential function corresponds to the single mutant G48V. The TI procedure was realized for each $\lambda$ in three steps: (a) charge removal from the $\mathrm{H} \alpha$ atom of Gly, (b) changing Gly into Val, and (c) charge 
Table 1. Summary of Major Findings in wt and Single Mutant Saquinavir-HIV-1 PR Complexes

\begin{tabular}{|c|c|c|c|c|c|c|c|}
\hline complexes & $\begin{array}{l}\text { average flap } \\
\text { RMSD }(\AA)\end{array}$ & $\begin{array}{l}\text { RMSD between SQV in mutated forms and its } \\
\text { structure in wt }(\AA)\end{array}$ & $\begin{array}{l}\text { no. of } \mathrm{H}- \\
\text { bonds }\end{array}$ & $\begin{array}{l}\text { water-mediated } \\
\text { interactions }\end{array}$ & $\begin{array}{l}\Delta G_{\mathrm{MM}-\mathrm{PBSA}} \\
(\mathrm{kcal} / \mathrm{mol})\end{array}$ & $\begin{array}{l}K_{i}^{\mathrm{MM}-\mathrm{PBSA}} \\
(\mathrm{nM})\end{array}$ & $K_{i}^{\exp }(\mathrm{nM})$ \\
\hline wt & 0.52 & & 7 & 2 & -12.25 & 2.5 & $0.42^{22}$ \\
\hline L10I & 0.99 & 1.67 & 5 & 2 & -11.53 & 7.4 & \\
\hline G48V & 0.55 & 3.16 & 3 & & -11.10 & 14.5 & $36^{22}$ \\
\hline L63P & 1.39 & 4.03 & 4 & 1 & -10.89 & 21 & \\
\hline A71V & 1.45 & 3.99 & 4 & 2 & -12.04 & 3.2 & \\
\hline G73S & 0.68 & 1.73 & 5 & 4 & -11.82 & 4.6 & \\
\hline V82A & 0.87 & 4.21 & 3 & & -11.76 & 5.1 & \\
\hline $\mathrm{I} 84 \mathrm{~V}$ & 0.86 & 1.29 & 3 & & -10.90 & 20.6 & \\
\hline
\end{tabular}

(a)

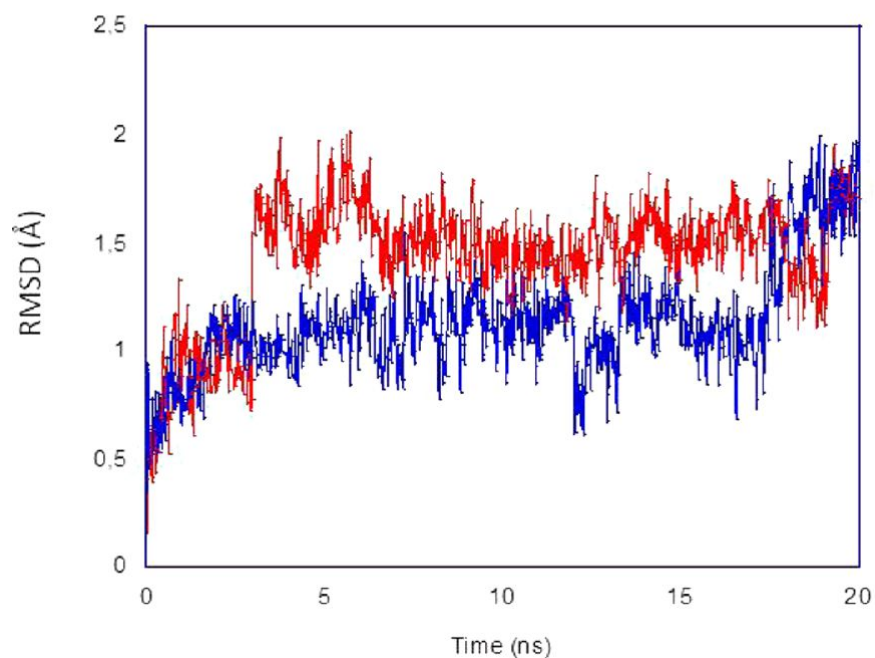

(b)

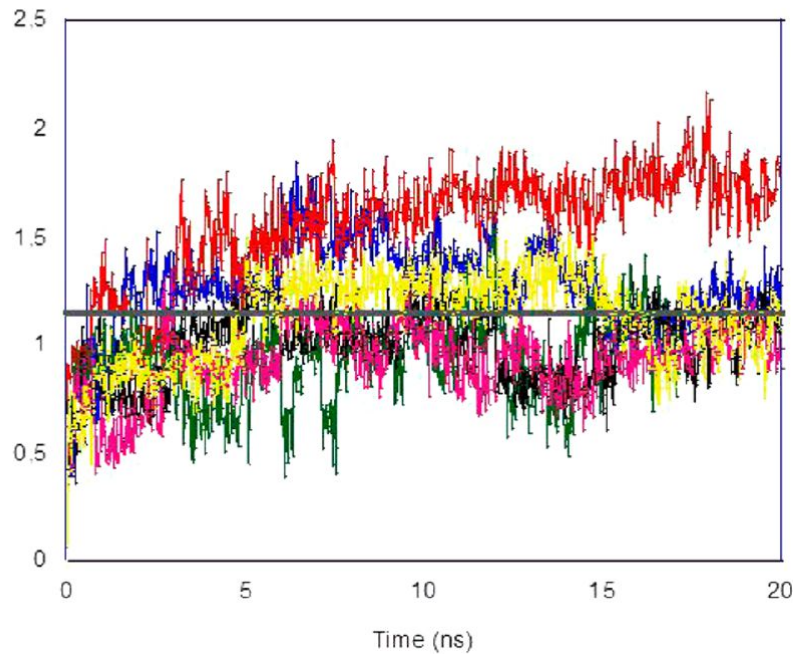

Figure 2. RMSD for saquinavir with respect to its initial conformation in HIV-1 PR complexes: (a) wt (blue) and G48V (red); (b) L63P (blue), A71V (red), V82A (green), G73S (black), I84V (purple), and L10I (yellow). The gray line shows the average RMSD for saquinavir in the wt complex. For clarity, individual RMSD plots for each mutant are presented in Figure S5.

addition on all side chain atoms of Val. Since the wt and mutant enzymes have different numbers of atoms due to the change in position 48 , we used soft-core potentials ${ }^{51}$ that were activated only during the second step, which involves the disappearance and appearance of atoms. MD simulations for the TI calculation were performed according to the protocol described above. The sampling space was defined by 12 different $\lambda$ values, and for each $\lambda$ we ran six (three steps for each wt and G48V) independent, 2 ns MD simulations. For our calculations, the values of $\lambda$ and their respective weights were taken from the AMBER 11 manual (Table S2).

\section{RESULTS}

The comparison of wt and mutant molecular trajectories showed the differences and similarities among structures, as summarized in Table 1. Despite its potency, saquinavir's continuous administration soon led to the emergence of drug resistant strains. Therefore, it is imperative to understand the behavior of the drug in the different PR mutants that have arisen due to its use.

III.1. Conformational Analysis of Saquinavir in the Binding Site of wt and Mutated Forms of HIV-1 PR. RMSD calculations for the C $\alpha$ atoms of HIV-1 PR in wt and all mutated complexes showed that the simulations converged after $\approx 10 \mathrm{~ns}$, thus yielding stable trajectories for all systems (Figure S1). The calculation of the RMSD may also provide information on the structure of the drug inside the protease: in
Figure 2, the RMSD for all atoms of the drug (compared to its initial conformation) in wt, G48V, L63P, A71V, V82A, G73S, $\mathrm{I} 84 \mathrm{~V}$, and $\mathrm{L} 10 \mathrm{I}$ are presented. The most prominent changes in saquinavir were observed in the A71V mutant, with the RMSD stabilizing to $\approx 1.8 \AA$ by the end of the simulation. In all other complexes, the structure of saquinavir does not present significant changes from the starting conformation, with values fluctuating around 1.2-1.5 $\AA$. The only exception is presented in the mutant strain V82A, where the inhibitor inside the active site shows the lowest RMSD during the simulation, with a mean value of $\approx 0.9 \AA$. This may attest that the particular mutation does not induce major changes in saquinavir throughout the simulation. Although the conformations of the drug inside the binding cavity of V82A resemble its starting structure, during the first half of the simulation, saquinavir appears more mobile in V82A than in any other mutant; however, it eventually stabilizes after $10 \mathrm{~ns}$. One may also note that saquinavir in G48V is slightly more flexible than when bound to the wt.

Clustering Analysis. Following the RMSD calculations of saquinavir inside the binding cavity of the enzyme, we performed a clustering analysis. Calculations have been carried out for the drug in wt and in all single mutant complexes. One common characteristic in all systems is the presence of only one representative conformation of the drug, as shown in Figure S2. This strongly suggests that saquinavir inside the 
(a)

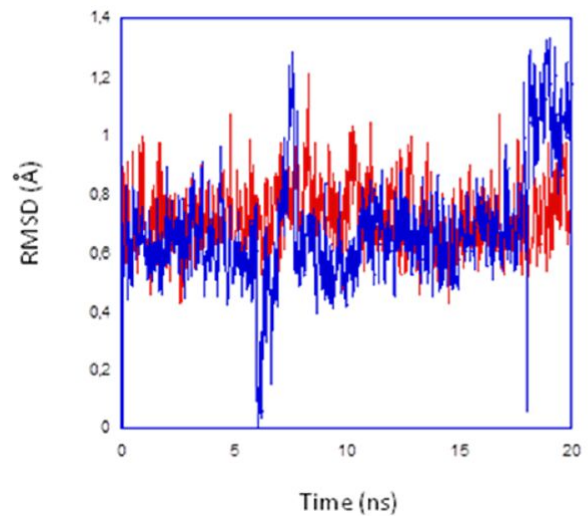

(b)

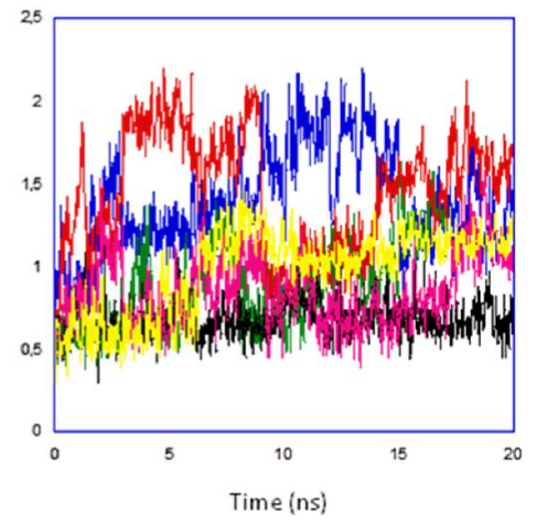

Figure 3. RMSD for the flap C $\alpha$ atoms (residues $45-55$ and $45^{\prime}-55^{\prime}$ ), with respect to their initial conformation in HIV-1 PR complexes: (a) wt (blue) and G48V (red); (b) L63P (blue), A71V (red), V82A (green), G73S (black), I84V (purple), and L10I (yellow). Individual plots for each mutant are shown in Figure S6.

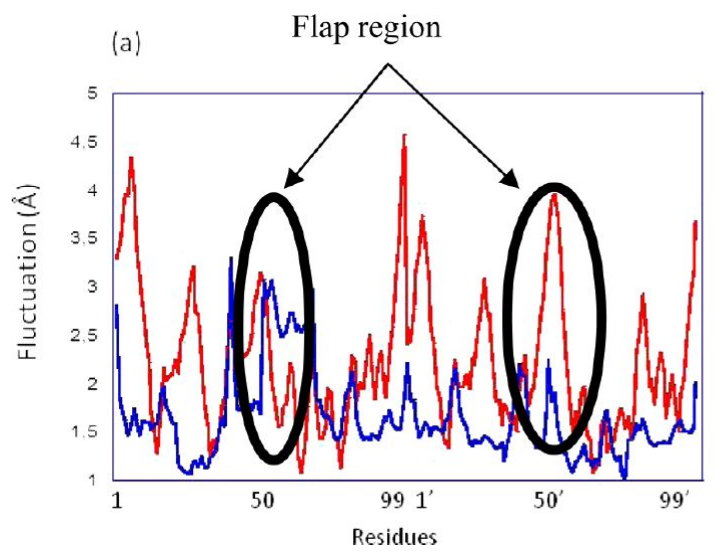

(b)

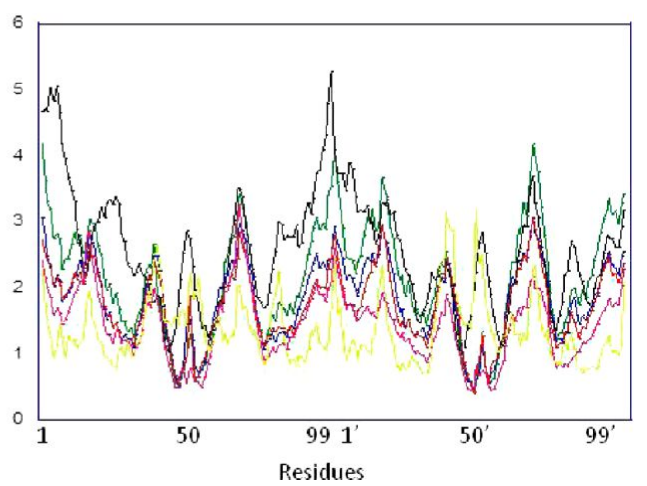

Figure 4. Atomic fluctuations for protease residues in the HIV-1 PR complexes: (a) wt (blue) and G48V (red); (b) L63P (blue), A71V (red), V82A (green), G73S (black), I84V (purple), and L10I (yellow). For clarity, individual plots for each mutant are shown in Figure S7.

binding cavity adopts an orientation that favors certain interactions between itself and the protease.

In wt HIV-1 PR, saquinavir adopts a slightly "bent" conformation (Figure S2) that may assist in favorable interactions with the protein. The MD representative structure of saquinavir resembles the crystal structure of the drug, with an RMSD between them of only $0.41 \AA$. Interestingly, saquinavir in the G73S mutant also resembles its structure in wt (Figure S2); the RMSD between the two conformations is $1.73 \AA$. Similarly, there is no large difference between saquinavir into wt and into the I84V and L10I single mutants that present RMSD values of $1.29 \AA$ and $1.67 \AA$, respectively.

Compared to saquinavir in wt, a structural difference was observed for its representative structure in the V82A mutant, where the RMSD between them is $4.21 \AA$; the representative conformation of saquinavir inside V82A adopts a more extended form. Similar structures were observed for the drug in L63P and A71V that have RMSD of $4.0 \AA$, compared to the wt. This conformational change in saquinavir may have contributed to the overall RMS change observed for the A71V complex in Figure 2b.

Another feature arising from the clustering analysis is the stability of the hydroisoquinoline group in saquinavir. The group shows the smallest conformational variation in all representative structures between wt and mutant strains. It was suggested that this moiety is less flexible than the quinoline group in saquinavir. In conclusion, however, saquinavir presents a stable conformation inside the active site cavity of the protease. This is of importance regarding the binding process and consequently the mechanism of inhibition.

III.2. Conformational Properties of the Flap Region. As observed in other experimental and theoretical studies, ligand binding is closely related with conformational changes on the flaps of the enzyme. ${ }^{12,13}$ This region is defined by residues 4455 and $44^{\prime}-55^{\prime}$, and has been implicated in the modulation of the entrance and trapping of ligands inside the catalytic site. Thus, following the analysis regarding the structure of the drug in the active site, it has been deemed important to consider the flap region in the different mutants.

In Figure 3, we have depicted the RMS deviations throughout the simulation for the flap region. It is important to note that the only mutant belonging to the flap region of the enzyme is G48V, with Gly48 being very close to the flap tip. Therefore, any mutations in this position are likely to induce changes in the overall structure of the protein. The analysis of the G48V trajectory compared to the wt showed minor changes in the flap region as depicted by the RMSD values (Figure 3a). However, it has been suggested in other studies that the change from Gly to a more hydrophobic residue such as Val could cause a specific structural change in the tip of the flap (Ile50). ${ }^{22,32}$ Namely, the flap tip in the G48V mutant has been shown to create a curl that subsequently affects the interaction 
Table 2. Principal Hydrogen Bonds in Saquinavir-HIV-1 PR Complexes ${ }^{a}$

\begin{tabular}{|c|c|c|c|c|c|c|}
\hline SQV-residue ${ }^{b}$ & wt & L10I & G48V & L63P & A71V & G73s \\
\hline Asp25 & 58 & $33 / 23$ & & & & 88 \\
\hline Thr26 & & & & 20 & 11 & 11 \\
\hline Asp25' & 45 & & 32 & 19 & 88 & \\
\hline Asp29' & & 95 & & & 13 & \\
\hline Gly48' & 81 & & & & & 60 \\
\hline Ile $50^{\prime}$ & & & 56 & & & \\
\hline Asp25- $\mathrm{H}_{2} \mathrm{O}-\mathrm{SQV}$ & & & & 20 & & \\
\hline Asp29- $\mathrm{H}_{2} \mathrm{O}-\mathrm{SQV}$ & 39 & 35 & & & 12 & 40 \\
\hline Ile $50^{\prime}-\mathrm{H}_{2} \mathrm{O}-\mathrm{SQV}$ & & & & & 36 & \\
\hline
\end{tabular}

${ }^{a}$ For detailed information regarding the HB interactions, see Table S3 in the Supporting Information. ${ }^{b}$ Occupancy is given as the percentage of simulation time that a specific interaction exists; interactions occurring less than $10 \%$ of the simulation time are not shown.

between flaps. Such a behavior was not observed in our case, since phi-psi plots for residues 48-51 revealed minor differences between wt and mutated complexes (Figure S3). The only exception was observed in Gly49 ( $\varphi$ angle) for the mutated form, which showed a slight deviation from the $\alpha$-helix wt structure. Interestingly, however, $\mathrm{C} \alpha$ atomic fluctuations of the protease residues in wt and G48V (Figure 4a) showed that the flap tips in G48V are more mobile than in wt. This indicates the absence of significant interactions between the two flaps of HIV-1 PR in the mutated form and consequently the less effective entrapment of the inhibitor inside the binding cavity.

Following this observation, we investigated the same region in the other single mutations. In all other mutants, the RMS deviations for the flap region were greater than the wt (Figure $3 \mathrm{~b})$. The only exception is G73S, which presented similar values to the wt. The most profound changes in the flaps of the protease came from mutations L63P and A71V. Indeed in these cases, the RMSD reached up to $2 \AA$ compared to the starting conformation. Furthermore, they occasionally return to lower values, thus suggesting that they probably alternate between "semi-open" and "closed" states several times during the simulation. However, their respective atomic fluctuations appear to be significantly low (Figure $4 \mathrm{~b}$ ) and comparable to the wt. This suggests that despite their conformational changes, the flaps are primarily stable during the simulation.

Extending our analysis to other parts of the protease, we observed that the terminal regions including residues 80-99 and $1^{\prime}-20^{\prime}$ appear increasingly mobile in most complexes. These residues are located at the interface between the two monomers of the protein. Many of the single mutations studied here are close to this area. Therefore, any changes in these residues may affect the stability of the dimer and consequently the activity of the enzyme. Although single mutations G48V, G73S, and L10I do not present any major conformational changes in the flap region (Figure $3 b$ ), the atomic fluctuations regarding the flap tip residues (Ile50/50') show greater mobility compared to all the other single mutants. Also, I84V shows similar mean RMS values with the single mutant G73S, both for the flap region and for saquinavir into the cavity (compare Figures 2 and 3). Although the amino acids in positions 73 and 84 are different, it may be hypothesized that any changes in the specific area do not lead to significant conformational changes in the enzyme.

III.3. Interactions Involving wt and Single Mutant Complexes. Following the conformational analysis, we next investigated the interactions between the protease (wt and mutants) and saquinavir. The hydrogen bonds (HBs) between the drug and the enzyme may provide information on the changes caused by the mutations and the behavior of saquinavir in different viral strains.

$\mathrm{HB}$ analysis of the MD trajectories revealed that saquinavir forms seven HB interactions with wt protease (Tables 2 and S3 and Figure 5a). The bent conformation of the drug (Figure S2) favors the formation of HBs that involve Gly48' at the flap of the protease, both aspartic acids in the catalytic site, and Arg8. This extensive network of interactions serves in anchoring saquinavir strongly into the binding cavity. The most frequent $\mathrm{HB}$ with Gly48' (being present for $81 \%$ of the simulation) is followed by another one with Asp25 (58\%); both bonds are dispersed throughout the simulation. Also, saquinavir forms three HBs with the catalytic Asp in position 25'. The presence of all these interactions may rationalize the existence of only one structural cluster for the drug in the wt (Figure S2). Finally, Arg8 presents an $\mathrm{HB}$ (29\%) that further strengthens the positioning of saquinavir into the binding cavity (Table S3). The interaction between saquinavir and Gly48' denotes the importance of the flap region in successfully trapping the ligand inside the protease. ${ }^{12,13}$

Our analysis revealed that saquinavir interacts also with five water molecules. Of these interactions, two are of particular interest (Tables 2, S3): two water molecules mediate HB interactions between saquinavir and Asp29 at the active site of the protease (Figure 5a). The involvement of water in direct HBs with the ligand may play a crucial role in the binding mechanism of the complexes.

Compared to the wt protease, the single mutant G48V presents a much less extensive $\mathrm{HB}$ network with saquinavir, with only three $\mathrm{HBs}$ between the inhibitor and the enzyme (Table S3, Figure 5b). Similarly to the wt, there is a direct interaction between the drug and amino acids on the catalytic site of the enzyme, where saquinavir forms a double $\mathrm{HB}$ with Asp25' (occurrence 32\%). However, the saquinavir-Gly48' interaction observed in the wt complex has been replaced by an HB between the drug and flap tip Ile50' (56\%, Figure 5b). In $\mathrm{G} 48 \mathrm{~V}$, the mutation from Gly to the more hydrophobic Val causes the loss (or diminishing) of certain interactions between saquinavir and protease: ${ }^{52}$ the saquinavir-Ile $50^{\prime} \mathrm{HB}$ in G48V is not as dominant compared to the saquinavir-Gly48' interaction in wt (Table 2). This change influences the stability of the flap region and at the same time also perturbs interactions inside the binding cavity that consequently lead to weaker binding of the drug to the protease. ${ }^{31}$ This may rationalize the role of $\mathrm{G} 48 \mathrm{~V}$ as a major mutation conferring resistance to saquinavir, as well as to other PIs (e.g., ritonavir 
(a)

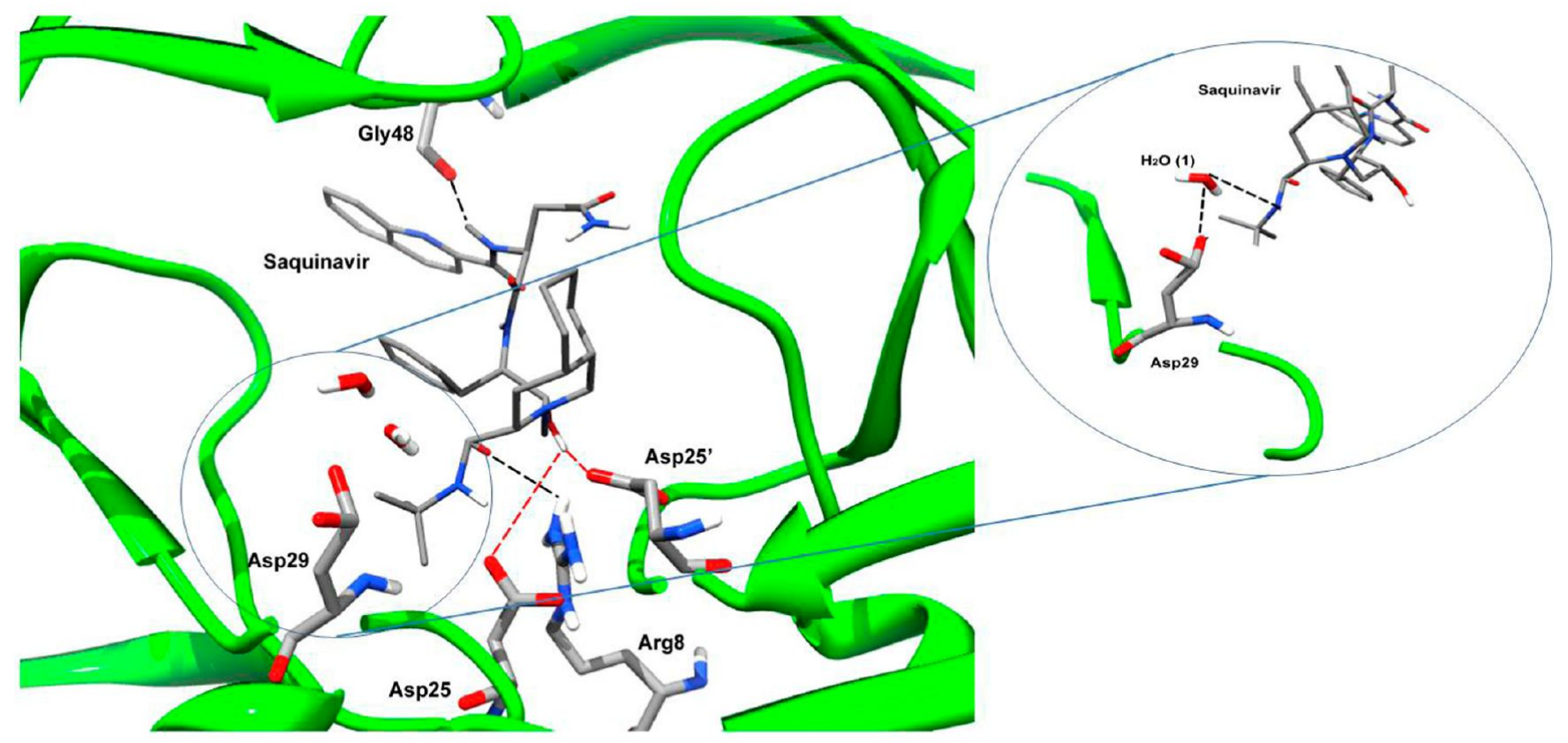

(b)

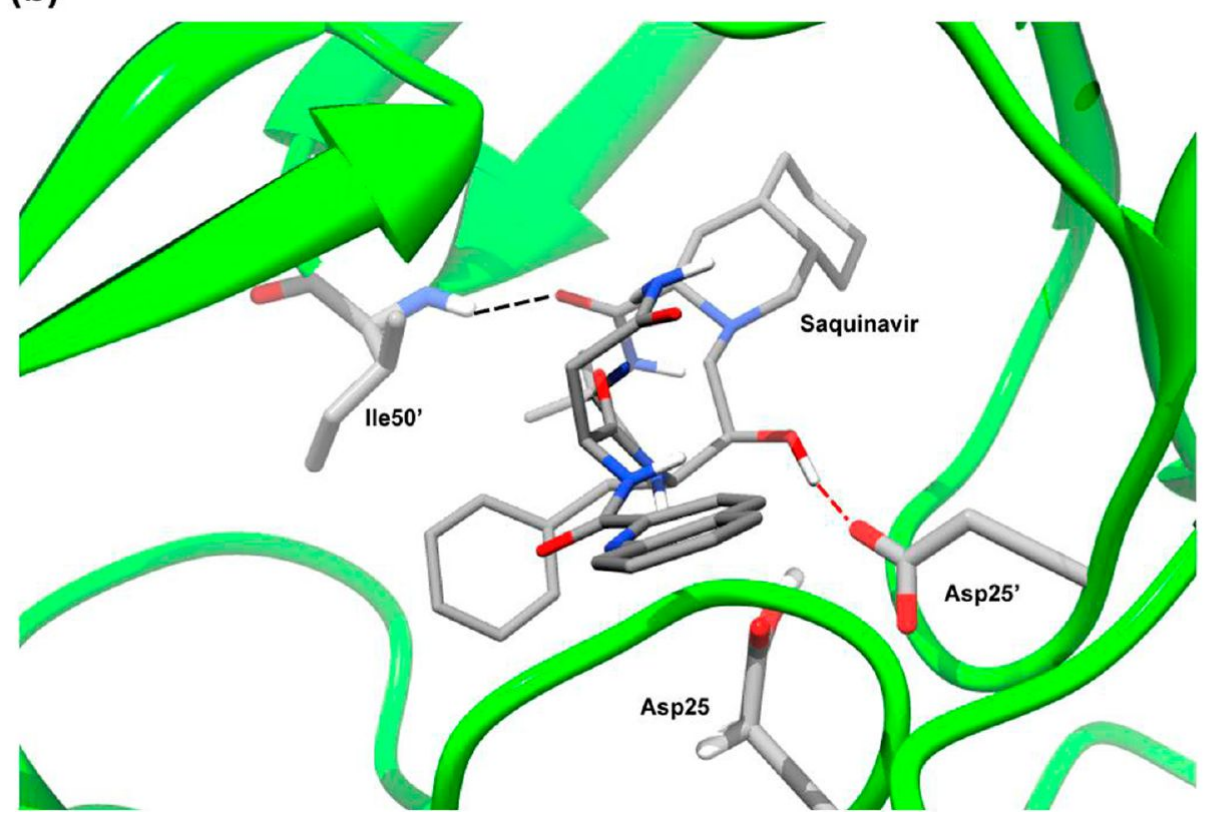

Figure 5. Main hydrogen bonds for saquinavir in complex with (a) HIV-1 $\mathrm{wt}_{\mathrm{t}} \mathrm{PR}$ and (b) HIV-1 $\mathrm{G}_{\mathrm{G} 48 \mathrm{~V}} \mathrm{PR}$. Red dotted lines denote interactions with catalytic aspartic acids at positions 25 and $25^{\prime}$. The side chains of amino acids participating in HBs are shown.

and atazanavir). ${ }^{21,53}$ Furthermore, water-mediated interactions between drug and enzyme are not present in the HIV-1 PRG48V complex.

HBs in all other single mutations are also diminished compared to the wt. A prevalent feature in L63P, V82A, A71V, and $\mathrm{I} 84 \mathrm{~V}$ single mutants is the absence of $\mathrm{HB}$ interactions between saquinavir and the flaps of the protease (Tables 2 and S3). Thus, one may deduce that the drug is not anchored strongly in the active site. The absence of interactions between flap residues and the drug may partially explain the decrease in the binding energy of saquinavir upon mutations. It may also be responsible for the greater structural changes of the single mutants compared to wt (Table 1). In L63P, saquinavir interacts only with residues at (or near) the active site (Thr26, Asp25', Asp30, and Arg8'). One interesting feature is that the interaction between Asp30 and saquinavir involves the aromatic ring of the drug ( $\pi$ interaction). Similar $\pi$ interactions were also present in the mutants L63P and A71V (Table S3).

As mentioned in other studies, ${ }^{54}$ water molecules affect ligand binding and also participate directly in the cleavage 
mechanism of the protease. ${ }^{35,55}$ Thus, we investigated possible protease-water-saquinavir interactions in all complexes, to find that they are present only in the L10I, L63P, A71V, and G73S strains. An interesting characteristic is that most water mediated HBs occur in the region of the active site (Asp25/ 29). Contrary to the other mutated complexes, in V82A, saquinavir forms two $\mathrm{HBs}$ with aspartic acids at positions $25^{\prime}$ and $29^{\prime}$ that are present throughout the simulation and hold saquinavir strongly attached at the active site of the protease (Table 2). This finding supports previous studies, which suggested that the V82A mutation is not conferring any major resistance to saquinavir. ${ }^{33}$

Interactions involving residues near or at the active site are present in all single mutants. Another common feature in wt and mutated forms is the HBs between saquinavir and Asp25/ $25^{\prime}$ and Thr26/26' in L10I, A71V, and G73S. Also, in G73S the interaction between the drug and Gly48' is also prevalent. In $\mathrm{A} 71 \mathrm{~V}$, although there is no direct interaction between the flap residues and the drug, there is a water mediated interaction between Ile50' and saquinavir. This highlights the importance of the active site residues and the flaps in anchoring the drug in opposite sides of the binding cavity, with direct implications toward effective saquinavir binding.

III.4. Intraflap and Interflap Interactions. The analysis of the $\mathrm{HB}$ interactions between saquinavir and the protease revealed the importance of the flaps in the ligand binding process. Therefore, we considered it extremely important to focus our interest in the flap region. ${ }^{48}$

As mentioned above, ${ }^{21} \mathrm{G} 48 \mathrm{~V}$ has been implicated as a major mutation conferring resistance to saquinavir. In Tables 3 and

Table 3. Flap-Flap HB Interactions in wt and G48V Saquinavir Complexes

\begin{tabular}{cccc} 
& & \multicolumn{2}{c}{ occupancy (\%) } \\
\cline { 3 - 4 } residue 1 & residue 2 & wt & G48V \\
Ile50' & Ile50 & 76 & \\
Ile50' & Gly52 & 26 & \multirow{2}{*}{42} \\
Ile50 & Gly51' & & \\
Ile50-water-Ile50' & 25 &
\end{tabular}

S4, we present the $\mathrm{HB}$ interactions involving the flaps of wt and the G48V mutant: it is evident that the flaps of the protein are stabilized in both complexes via significant interflap and intraflap interactions; however, interflap $\mathrm{HBs}$ are significantly more dominant in the wt.

Of major importance was the presence of a water molecule between the flap tips (Ile50 and Ile50') of the enzyme in the saquinavir-wt complex (Table 3). This is in accordance with other studies ${ }^{56-58}$ that have explored the role of water in different HIV-1 PR systems. The mutation in position 48 from Gly to Val causes a weakening of the interactions between the two flaps of the protease. In the wt complex, the Ile50-Ile50' $\mathrm{HB}$ has an occurrence of $76 \%$, while in the G48V mutant this interaction is absent. This indicates that the particular mutation causes conformational changes in the vicinity of the flaps, as being hypothesized above. The more hydrophobic chain of $\mathrm{Val}$ compared to Gly leads to structural changes in the neighboring amino acids, which in turn may affect the binding of saquinavir. This observation is further supported by structural studies on the double mutant strain G48V/L90 M that show the effect of the flap mutation in the conformation of the flap region. ${ }^{52}$ The crystal structure of the double mutant has provided information on the importance of Gly48: it has been suggested that the change from Gly to Val leads to a significant loss of interactions between saquinavir and the protease. ${ }^{53}$ Similarly, we observed that mutation $\mathrm{G} 48 \mathrm{~V}$ has greatly impacted the interflap interaction potential by abolishing interactions between Ile $50^{\prime}$ and Ile50, as well as between Ile50' and Gly52 (Table 3). Also, saquinavir in the G48V interacts less effectively (compared to the wt) with the flap via an HB with Ile50' (56\%, Table 2). This may have induced changes in interflap interactions leading to the replacement of the Ile 50 '-Ile $50 \mathrm{HB}$ (present in wt) with a less frequent interaction between Ile50 and Gly51' (42\%). Compared with the previous changes conferred by the G48V mutation in the flaps, it is not surprising that viral strains with the particular mutation show increased resistance to saquinavir.

The relation of Gly48 to the flap stability is also depicted by the atomic fluctuations of the flap residues in Figure 4. The conformational changes caused by the mutation, together with the interactions that form or disappear in the two complexes, greatly increase the mobility of the flap region in $\mathrm{G} 48 \mathrm{~V}$ mutant.

Table 4. Energetic analysis for saquinavir-HIV1-PR complexes as obtained by MM-PBSA and TI calculations

\begin{tabular}{|c|c|c|c|c|c|c|c|c|}
\hline \multirow[b]{2}{*}{ energy $^{a}(\mathrm{kcal} / \mathrm{mol})$} & \multicolumn{8}{|c|}{ saquinavir-HIV-1 PR } \\
\hline & wt & L10I & G48V & L63P & A71V & G73S & V82A & I $84 \mathrm{~V}$ \\
\hline$\Delta E_{\text {elec }}$ & -48.35 & -47.45 & -45.54 & -45.18 & -47.51 & -46.53 & -43.80 & -45.04 \\
\hline$\Delta E_{\mathrm{vdw}}$ & -52.18 & -51.89 & -55.22 & -49.47 & -50.67 & -52.74 & -54.23 & -55.69 \\
\hline$\Delta E_{\mathrm{MM}}$ & -100.53 & -99.34 & -100.76 & -94.65 & -98.18 & -99.27 & -98.03 & -100.73 \\
\hline$\Delta G_{\mathrm{NP}}$ & -9.35 & -9.80 & -10.01 & -9.65 & -8.74 & -10.12 & -9.04 & -9.77 \\
\hline$\Delta G_{\text {ele }}$ & 74.29 & 72.97 & 75.18 & 71.27 & 71.47 & 74.87 & 70.51 & 74.24 \\
\hline$\Delta G_{\text {solv }}$ & 64.94 & 63.17 & 65.17 & 61.62 & 62.73 & 64.75 & 61.46 & 64.47 \\
\hline$\Delta H_{(\mathrm{MM}+\text { solv })}$ & -35.59 & -36.17 & -35.59 & -33.03 & -35.45 & -34.52 & -36.63 & -36.26 \\
\hline$-T \Delta S_{\text {tot }}$ & 23.34 & 24.64 & 24.49 & 22.14 & 23.41 & 22.70 & 24.87 & 25.36 \\
\hline$\Delta G_{\text {bind }}$ MM-PBSA & -12.25 & -11.53 & -11.10 & -10.89 & -12.04 & -11.82 & -11.76 & -10.90 \\
\hline$K_{i}(\mathrm{nM})^{b}$ & 2.5 & 7.4 & 14.5 & 21 & 3.2 & 4.6 & 5.1 & 20.6 \\
\hline$\Delta \Delta G_{\mathrm{TI}}$ & & & 0.28 & & & & & \\
\hline$\Delta \Delta G_{\text {MMPBSA }}$ & & 0.72 & 1.15 & 1.36 & 0.21 & 0.43 & 0.49 & 1.35 \\
\hline$\Delta \Delta G_{\exp }$ & & & 2.65 & & & & & \\
\hline$K_{\text {iexp }}(\mathrm{nM})$ & $0.42^{c}$ & & $36^{c}$ & & & & & \\
\hline
\end{tabular}

${ }^{a}$ The standard deviation values for the energy calculations are presented in Table S4. ${ }^{b}$ The $K_{i}$ values were calculated based on the equation $\Delta G=\mathrm{RT}$ $\ln K_{i} \cdot{ }^{c}$ See ref 22 . 
III.5. Energetic Analysis. The MM-PBSA results revealed the impact of mutations on the binding affinity of saquinavir to HIV-1 PR (Table 4). Enthalpy and entropy plots over time for all HIV-1 PR complexes showed that the binding energy calculations converged after approximately $10 \mathrm{~ns}$ (Figure S4).

Our calculations supported $\mathrm{G} 48 \mathrm{~V}$ being a major mutation, as it has a noticeable impact compared to the wt $\left(\Delta \Delta G_{\mathrm{MM}-\mathrm{PBSA}}=\right.$ $1.15 \mathrm{kcal} / \mathrm{mol}$ ). Similarly, the MM-PBSA calculations showed that single mutations $184 \mathrm{~V}$ and $\mathrm{L} 63 \mathrm{P}$ reduce the binding affinity significantly, by approximately $1.35 \mathrm{kcal} / \mathrm{mol}$. Both mutations involve changes from long side chains (Ile and Leu) to shorter (Val) or more rigid (Pro) amino acids. Ile84 is positioned inside the binding cavity, and we notice that a change to an amino acid with a shorter side chain (Val) may lead to the loss of several $\mathrm{HB}$ interactions (Table 2). By decreasing the potential for $\mathrm{HB}$ formation inside the binding cavity, the inhibitor may not be effectively bound. On the other hand, the L63P mutation did not affect the binding of the ligand directly, since this amino acid is located away from the binding cleft. It is part of a $\beta$-sheet conformation that supports the flaps of the enzyme (Figure 1). Changes in this position may destabilize the conformation of the flaps and indirectly reduce ligand binding.

The lowest impact was observed in the single mutant A71V, with a binding energy of $-12.04 \mathrm{kcal} / \mathrm{mol}\left(\Delta \Delta G_{\mathrm{MM}-\mathrm{PBSA}}=\right.$ $0.21 \mathrm{kcal} / \mathrm{mol}$ ). This could be partially attributed to the close structural relationship between $\mathrm{Ala}$ and $\mathrm{Val}$, since they have similar size and both are hydrophobic; their similar nature may have resulted in the preservation of $\mathrm{HBs}$ in $\mathrm{A} 71 \mathrm{~V}$ and in the presence of water bridges, as shown in Table 2. Thus, it may be hypothesized that this mutation does not readily affect the binding of the drug.

Single mutants L10I and V82A belong to the binding cavity and alter directly the interactions of the drug with the protease. This can be explained by first considering the extensive $\mathrm{HB}$ network in wt HIV-1 PR: the presence of HBs on two opposite sides of the cavity-Gly48' (flap) and Asp25 (active site)may readily justify the high inhibitory effect of saquinavir. However, this trend is not observed to a significant extent in any of these single mutants. Thus, it could partially rationalize the decrease in the binding affinity of saquinavir.

Mutations (e.g., V82A, I84V) that are close to the interface of the two monomers of HIV-1 PR (two $\alpha$-helices that support the binding cleft residues $\left.84-94 / 84^{\prime}-94^{\prime}\right)^{16}$ affect the interactions between them and consequently lead to decreased functionality of the enzyme. However, the two permanent HBs-with Asp25' and Asp29'-in V82A may justify that the calculated binding affinities $\left(K_{i}\right)$ between wt and V82A show very small differences ( $2.5 \mathrm{nM}$ and $5.1 \mathrm{nM}$, respectively). This is not surprising, since only in patients that have undergone prolonged treatment with saquinavir did the V82A mutation confer resistance, and usually in conjunction with the mutation G48V. ${ }^{21,54}$ However, mutation V82A is important in developing resistance to other drugs, such as indinavir and ritonavir. ${ }^{59}$

The MM-PBSA analysis also offers individual contributions to the binding energy (Table 4). A general feature is that they are fairly similar in all mutations with the van der Waals interactions being the most prominent, closely followed by the electrostatic contributions to the total binding energy. Also, the nonpolar contribution to solvation is a significant component, mostly in G48V and G73S complexes.

The impact of a mutation to the binding affinity of saquinavir can be further elucidated by calculating the enthalpy $(\Delta H)$ contributions of individual amino acids. A comparison between the wt protease and the G48V mutant is presented in Figure 6.

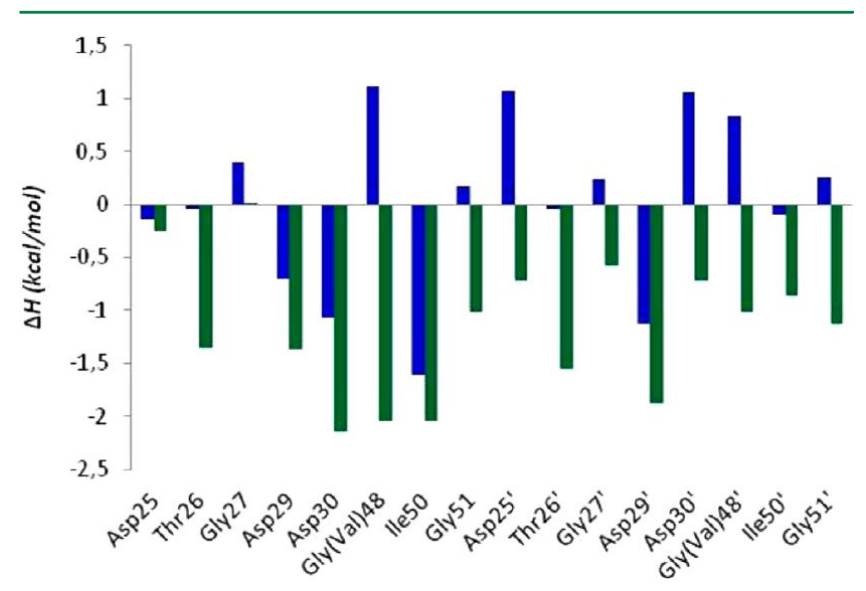

Figure 6. Per residue contributions to $\Delta H$ for saquinavir complexes HIV- $1_{\text {wt }}$ PR (green) and HIV-1 $1_{\mathrm{G} 48 \mathrm{~V}}$ PR (blue). Individual contributions for active site and flap residues are displayed.

The graph shows the energy contributions of important residues (active site and flaps). In the wt, all selected amino acids had favorable contributions ranging from -0.1 to -2.2 $\mathrm{kcal} / \mathrm{mol}$, whereas in the $\mathrm{G} 48 \mathrm{~V}$ mutant, residues at the active site (Asp25' and Gly27/27') and the flaps (Val48/48' and Gly51/51') show unfavorable contributions. Interestingly, the greatest loss in binding enthalpy is due to mutated flap residues 48 and $48^{\prime}$. It is worth mentioning that although active site residues, such as Thr26/26' and Gly27/27', did not participate in HBs with saquinavir, they favored inhibitor's binding into wt, while they displayed a minor or negative contribution to the binding energy in the mutated complex. Despite the preservation of the $\mathrm{HB}$ between Asp25' and saquinavir in the G48V mutant, the contribution to the binding energy for the specific residue is still unfavorable. This suggests that the mutation causes structural rearrangements to the binding cavity leading to a decreased binding affinity.

Besides enthalpy, the changes observed in the conformational entropy of a complex greatly affect the binding affinity, and failure to take them into account may lead to overestimations in the energy profile of the system. ${ }^{60}$ It has been shown that in certain PIs, such as saquinavir, the entropic component has a great impact on their binding. ${ }^{31}$ Our calculations (Table 4) show similar patterns regarding the total conformational entropy with previous studies. ${ }^{23,60}$ In G48V, the contribution of enthalpy was not affected by the mutation $\left(\Delta H_{\mathrm{wt}}=\Delta H_{\mathrm{G} 48 \mathrm{~V}}\right)$; upon $\mathrm{G} 48 \mathrm{~V}$ mutation, the favorable van der Waals change $\left(\Delta E_{\mathrm{vdW}, \mathrm{G} 48 \mathrm{~V}}=-55.22 \mathrm{kcal} /\right.$ mol, $\left.\Delta E_{\mathrm{vdW}, \mathrm{wt}}=-52.18 \mathrm{kcal} / \mathrm{mol}\right)$ was balanced with the unfavorable change in electrostatic interactions $\left(\Delta E_{\text {elec, } \mathrm{G} 48 \mathrm{~V}}=\right.$ $\left.-45.54 \mathrm{kcal} / \mathrm{mol}, \Delta E_{\text {elec,wt }}=-48.35 \mathrm{kcal} / \mathrm{mol}\right)$ to maintain $\Delta H$ practically fixed. Thus, the loss in binding energy for G48V is solely attributed to the change in entropy. Furthermore, it was observed that the vibrational entropy for G48V versus the wt is unfavorable by $\approx 1 \mathrm{kcal} / \mathrm{mol}\left(-T \Delta S_{\mathrm{vib}, \mathrm{G} 48 \mathrm{~V}}=3.87 \mathrm{kcal} / \mathrm{mol}\right.$ and $-T \Delta S_{\text {vib,wt }}=2.98 \mathrm{kcal} / \mathrm{mol}$, Table S6), in accordance with previous observations. ${ }^{31}$

To reach a higher level of accuracy, the TI method has been employed for the calculation of the relative free energy of binding, between the wt and G48V complexes. Negative (or positive) values of $\Delta \Delta G_{\mathrm{TI}}$ denote whether the mutation is a 
favorable (or unfavorable) process. In this case, the estimated value for $\Delta \Delta G_{\mathrm{TI}}$ is $0.28 \mathrm{kcal} / \mathrm{mol}$. This shows that the amino acid change from Gly to Val negatively impacted saquinavir's binding affinity. This, in combination with the results obtained from the conformational and MM-PBSA analyses further strengthens the importance of the flap region in successfully trapping saquinavir inside the binding cavity. In agreement with previous experimental evidence, the value of the free energy change between the two states was calculated to be slightly positive, as a single mutation is expected to confer low level resistance. ${ }^{15,16,22}$

\section{CONCLUSIONS}

The conformational properties and binding modes of eight saquinavir-HIV-1 PR complexes in the wild-type and single mutant forms, L10I, G48V, L63P, A71V, G73S, V82A, and $\mathrm{I} 84 \mathrm{~V}$, have been investigated by means of $\mathrm{MD}$ simulations and binding free-energy (MM-PBSA and TI) calculations. Binding energy analysis showed that mutations G48V, L63P, and I84V significantly deteriorate saquinavir binding compared to the wild-type and other mutated complexes. In contrast, A71V had a negligible effect on the binding affinity of saquinavir. An extensive comparison of structural properties, energetics, and dynamics for all systems indicated the main reasons that particular mutations confer resistance to saquinavir: (1) Mutations that induce flexibility to the flaps of the protease (G48V, L63P, L10I, G73S) lead to the reduction of binding. Conversions of the flaps between semiopen and closed states may be observed (A71V) without significant loss in binding affinity, as long as the flaps remain primarily stable. (2) Conformational changes of saquinavir may not affect binding (V82A, A71V), provided that its structure eventually stabilizes, as observed in the wt. The hydroisoquinoline group of saquinavir appeared particularly stable in all systems; saquinavir structures in all mutants adopted specific conformations that favor certain interactions: (3) Saquinavir-related $\mathrm{HB}$ interactions with the active site (Asp25/25'/29/29'/30/30') and the flaps of HIV-1 PR should be present to hold the drug into the binding cavity in a compact conformation. Mutations that result in major resistance (G48V, L63P, I84V) do not preserve favorable interactions with the active site and the flaps. (4) A water-mediated $\mathrm{HB}$ interaction between saquinavir and the region of the active site (Asp29) is of major importance (wildtype, A71V, G73S, L63P, L10I). Mutants that lack this interaction (e.g., G48V, I84V) showed significantly lower (experimental and theoretical) binding affinities. (5) Enhanced van der Waals interactions and electrostatics are the main contributions to the binding energy, with similar values among mutants $\left(-55.7 \mathrm{kcal} / \mathrm{mol}<\Delta E_{\mathrm{vdW}}<-49.5 \mathrm{kcal} / \mathrm{mol},-48.4\right.$ $\left.\mathrm{kcal} / \mathrm{mol}<\Delta E_{\text {elec }}<-43.8 \mathrm{kcal} / \mathrm{mol}\right)$. This work constitutes a computational prediction of HIV-1 PR drug resistance.

\section{ASSOCIATED CONTENT}

\section{S Supporting Information}

Figures showing the RMSD of HIV-1 PR, saquinavir, and the flap region in all complexes; Ramachadran plots for wt/G48V; MM-PBSA convergence plots; fluctuations; and representative structures of SQV in all complexes. Tables showing amino acid substitutions, $\lambda$ values for TI, HB interactions between SQV and protease in all complexes, intra- and interflap HBs, binding energies with standard deviations, and the entropy decomposition for wt and G48V. This material is available free of charge via the Internet at http://pubs.acs.org.

\section{AUTHOR INFORMATION}

\section{Corresponding Author}

*E-mail: tzoupis@eie.gr (H.T.), gleonis@eie.gr (G.L.), mpapad@eie.gr (M.G.P.).

Notes

The authors declare no competing financial interest.

\section{ACKNOWLEDGMENTS}

This research has been cofinanced by the European Union (European Social Fund-ESF) and Greek national funds through the Operational Program "Education and Lifelong Learning" of the National Strategic Reference Framework (NSRF)-Research Funding Program: Heracleitus II-Investing in knowledge society through the European Social Fund. Funding provided by the European Commission for the FP7REGPOT-2009-1 Project "ARCADE” (Grant Agreement No. 245866) is also acknowledged.

\section{REFERENCES}

(1) UNAIDS. http://www.unaids.org/en/ (accessed September 9, 2012).

(2) For Women > HIV and AIDS--Medicines to Help You. http:// www.fda.gov/ForConsumers/ByAudience/ForWomen/ucm118597. htm (accessed November 20, 2012).

(3) Mastrolorenzo, A.; Rusconi, S.; Scozzafava, A.; Barbaro, G.; Supuran, C. T. Curr. Med. Chem. 2007, 14, 2734-2748.

(4) Barbaro, G.; Scozzafava, A.; Mastrolorenzo, A.; Supuran, C. T. Curr. Pharm. Des. 2005, 11, 1805-1843.

(5) Hammer, S. M.; Squires, K. E.; Hughes, M. D.; Grimes, J. M.; Demeter, L. M.; Scurrier, J. S.; Eron, J. J., Jr.; Feinberg, J. E.; Balfour, H. H., Jr.; Deyton, L. R.; Chodakewitz, J. A.; Fischl, M. A. N Engl J Med 1997, 337, 725-733.

(6) Palella, F. J., Jr.; Delaney, K. M.; Moorman, A. C.; Loveless, M. O.; Fuhrer, J.; Satten, G. A.; Aschman, D. J.; Holmberg, S. D. N. Engl. J. Med. 1998, 338, 853-860.

(7) Imamichi, T. Front. Med. Chem. 2006, 3, 23-44.

(8) Coffin, J. M. Science 1995, 267, 483-489.

(9) Nicholson, L. K.; Yamazaki, T.; Torchia, D. A.; Grzesiek, S.; Bax, A.; Stahl, S. J.; Kaufman, J. D.; Wingfield, P. T.; Lam, P. Y.; Jadhav, P. K.; et al. Nat. Struct. Biol. 1995, 2, 274-280.

(10) Freedberg, D. I.; Ishima, R.; Jacob, J.; Wang, Y. X.; Kustanovich, I.; Louis, J. M.; Torchia, D. A. Protein Sci. 2002, 11, 221-232.

(11) Lapatto, R; Blundell, T.; Hemmings, A.; Overington, J.; Wilderspin, A.; Wood, S.; Merson, J. R.; Whittle, P. J.; Danley, D. E.; Geoghegan, K. F.; et al. Nature 1989, 342, 299-302.

(12) Hornak, V.; Okur, A.; Rizzo, R. C.; Simmerling, C. Proc. Natl. Acad. Sci. U. S. A. 2006, 103, 915-920.

(13) Hornak, V.; Okur, A.; Rizzo, R. C.; Simmerling, C. J. Am. Chem. Soc. 2006, 128, 2812-2813.

(14) Vondrasek, J.; Wlodawer, A. Proteins 2002, 49, 429-431.

(15) Gu, Z.; Gao, Q.; Fang, H.; Salomon, H.; Parniak, M. A.; Goldberg, E.; Cameron, J.; Wainberg, M. A. Antimicrob. Agents Chemother. 1994, 38, 275-281.

(16) Baldwin, E. T.; Bhat, T. N.; Liu, B.; Pattabiraman, N.; Erickson, J. W. Nat. Struct. Biol. 1995, 2, 244-249.

(17) Dandache, S.; Sevigny, G.; Yelle, J.; Stranix, B. R.; Parkin, N.; Schapiro, J. M.; Wainberg, M. A.; Wu, J. J. Antimicrob. Agents Chemother. 2007, 51, 4036-4043.

(18) King, J. R.; Wynn, H.; Brundage, R.; Acosta, E. P. Clin. Pharmacokinet. 2004, 43, 291-310.

(19) Vyas, S. P.; Sihorkar, V.; Jain, S. Int. J. Pharm. 2007, 330, 6-13.

(20) Bergersen, B. M.; Sandvik, L.; Dunlop, O.; Birkeland, K.; Bruun, J. N. Eur. J. Clin. Microbiol. Infect. Dis. 2003, 22, 731-763.

(21) Johnson, V. A.; Brun-Vezinet, F.; Clotet, B.; Gunthard, H. F.; Kuritzkes, D. R.; Pillay, D.; Schapiro, J. M.; Richman, D. D. Top. HIV Med. 2009, 17, 138-145. 
(22) Liu, F.; Kovalevsky, A. Y.; Tie, Y.; Ghosh, A. K.; Harrison, R. W.; Weber, I. T. J. Mol. Biol. 2008, 381, 102-115.

(23) Ode, H.; Neya, S.; Hata, M.; Sugiura, W.; Hoshino, T. J. Am. Chem. Soc. 2006, 128, 7887-7895.

(24) Toth, G.; Borics, A. J. Mol. Graphics Model. 2006, 24, 465-474.

(25) Hou, T.; McLaughlin, W. A.; Wang, W. Proteins 2008, 71, $1163-1174$.

(26) Kovalevsky, A. Y.; Louis, J. M.; Aniana, A.; Ghosh, A. K.; Weber, I. T. J. Mol. Biol. 2008, 384, 178-192.

(27) Kovalevsky, A. Y.; Tie, Y.; Liu, F.; Boross, P. I.; Wang, Y. F.; Leshchenko, S.; Ghosh, A. K.; Harrison, R. W.; Weber, I. T. J. Med. Chem. 2006, 49, 1379-1387.

(28) Jorgensen, W. L. Science 2004, 303, 1813-1818.

(29) McCammon, J. A. Curr. Opin. Struct. Biol. 1998, 8, 245-249.

(30) RCSB Protein Data Bank. http://www.rcsb.org/pdb/home/ home.do (accessed March 12, 2012).

(31) Stoica, I.; Sadiq, S. K.; Coveney, P. V. J. Am. Chem. Soc. 2008, 130, 2639-2648.

(32) Saen-oon, S.; Aruksakunwong, O.; Wittayanarakul, K.; Sompornpisut, P.; Hannongbua, S. J. Mol. Graphics Model. 2007, 26, $720-727$.

(33) Tie, Y.; Kovalevsky, A. Y.; Boross, P.; Wang, Y. F.; Ghosh, A. K.; Tozser, J.; Harrison, R. W.; Weber, I. T. Proteins 2007, 67, 232-242.

(34) Hyland, L. J.; Tomaszek, T. A., Jr.; Meek, T. D. Biochemistry 1991, 30, 8454-8463.

(35) Pietrucci, F.; Marinelli, F.; Carloni, P.; Laio, A. J. Am. Chem. Soc. 2009, 131, 11811-11818.

(36) Case, D. A.; Darden, T. A.; Cheatham, I. T. E.; Simmerling, C.; Wang, J.; Duke, R. E.; Luo, R.; Walker, R. C.; Zhang, W.; Merz, K. M.; Roberts, B. P.; Wang, B.; Hayik, S.; Roitberg, A.; Seabra, G.; Kolossvary, I.; Wong, K. F.; Paesani, F.; Vanicek, J.; Liu, J.; Wu, X.; Brozell, S. R.; Steinbrecher, T.; Gohlke, H.; Cai, Q.; Ye, X.; Wang, J.; Hsieh, M. J.; Cui, G.; Roe, D. R.; Mathews, D. H.; Seetin, M. G.; Sagui, C.; Babin, V.; Luchko, T.; Gusarov, S.; Kovalenko, A.; Kollman, P. A. AMBER 11; University of California: San Francisco, CA, 2010.

(37) Hornak, V.; Abel, R.; Okur, A.; Strockbine, B.; Roitberg, A.; Simmerling, C. Proteins 2006, 65, 712-725.

(38) Wang, J.; Wolf, R. M.; Caldwell, J. W.; Kollman, P. A.; Case, D. A. J. Comput. Chem. 2004, 25, 1157-1174.

(39) Jakalian, A.; Jack, D. B.; Bayly, C. I. J. Comput. Chem. 2002, 23, $1623-1641$.

(40) Jorgensen, W. L.; Madura, J. D.; Impey, R. W.; Klein, M. L. J. Chem. Phys. 1983, 79, 926-935.

(41) Izaguirre, J. A.; Catarello, D. P.; Wozniak, J. M.; Skeel, R. D. J. Chem. Phys. 2001, 114, 2090-2098.

(42) Ryckaert, J. P.; Ciccotti, G.; Berendsen, H. J. C. J. Comput. Phys. 1977, 23, 327-341.

(43) Honig, B.; Nicholls, A. Science 1995, 268, 1144-1149.

(44) Wang, W.; Kollman, P. A. J. Mol. Biol. 2000, 303, 567-582.

(45) Weiser, J.; Shenkin, P. S.; Still, W. C. J. Comput. Chem. 1999, 20, 217-230.

(46) Reyes, C. M.; Kollman, P. A. J. Mol. Biol. 2000, 297, 1145-1158.

(47) Hou, T.; Wang, J.; Li, Y.; Wang, W. J. Chem. Inf. Model. 2011, $51,69-82$.

(48) Cai, Y.; Schiffer, C. A. J. Chem. Theory Comput. 2010, 6, 13581368.

(49) Radmer, R. J.; Kollman, P. A. J. Comput.-Aided Mol. Des. 1998, $12,215-227$.

(50) Lawrenz, M.; Baron, R.; McCammon, J. A. J. Chem. Theory Comput. 2009, 5, 1106-1116.

(51) Beutler, T. C.; Mark, A. E.; van Schaik, R. C.; Gerber, P. R.; van Gunsteren, W. F. Chem. Phys. Lett. 1994, 222, 529-539.

(52) Hong, L.; Zhang, X. C.; Hartsuck, J. A.; Tang, J. Protein Sci. 2000, 9, 1898-1904.

(53) Fengling, L.; Kovalevsky, A. Y.; Ghosh, A. K.; Harrison, R. W.; Weber, I. T. J. Mol. Biol. 2008, 381, 102-115.

(54) Weber, I. T.; Agniswamy, J. Viruses 2009, 1, 1110-1136.
(55) Coates, L.; Tuan, H. F.; Tomanicek, S.; Kovalevsky, A.; Mustyakimov, M.; Erskine, P.; Cooper, J. J. Am. Chem. Soc. 2008, 130, 7235-7237.

(56) Okimoto, N.; Tsukui, T.; Kitayama, K.; Hata, M.; Hoshino, T.; Tsuda, M. J. Am. Chem. Soc. 2000, 122, 5613-5622.

(57) Leonis, G.; Czyznikowska, Z.; Megariotis, G.; Reis, H.; Papadopoulos, M. G. J. Chem. Inf. Model. 2012, 52, 1542-1558.

(58) Tzoupis, H.; Leonis, G.; Megariotis, G.; Supuran, C. T.; Mavromoustakos, T.; Papadopoulos, M. G. J. Med. Chem. 2012, 55, 5784-5796.

(59) Tie, Y.; Boross, P. I.; Wang, Y. F.; Gaddis, L.; Hussain, A. K.; Leshchenko, S.; Ghosh, A. K.; Louis, J. M.; Harrison, R. W.; Weber, I T. J. Mol. Biol. 2004, 338, 341-352.

(60) Chang, C. E.; Chen, W.; Gilson, M. K. Proc. Natl. Acad. Sci. U. S. A. 2007, 104, 1534-1539. 